\title{
INTERPRETACIÓN PATRIMONIAL: GESTIÓN EDUCATIVA Y LÚDICA PARA LA VALORACIÓN DEL LEGADO NATURAL Y CULTURAL DEL PARQUE LA ALAMEDA
}

\author{
HERITAGE INTERPRETATION: EDUCATIONAL AND RECREATIONAL \\ MANAGEMENT FOR THE VALUATION OF THE NATURAL AND \\ CULTURAL LEGACY OF THE ALAMEDA PARK
}

\begin{abstract}
Alexandra Araguillín Rojas
Pontificia Universidad Católica del Ecuador, Ecuador

Renato Cevallos Becerra

Universidad UTE, Quito, Ecuador

Aceptado: 10 de enero de 2020
\end{abstract}

\section{RESUMEN}

El Parque La Alameda es el parque más antiguo de la ciudad de Quito; es parte del área de protección patrimonial que tiene una variedad de atractivos naturales y culturales tangibles e intangibles de categorización patrimonial y emblemática que forman parte de la identidad del quiteño. Sin embargo, la escasa información y actividades recreativas-educativas relacionadas con sus atractivos lo ha convertido en un parque de paso. Por ello, el diseño de un programa interpretativo con actividades recreativas-educativas es una herramienta de gestión para la valoración y conservación del lugar y de difusión del turismo cultural.

En el proceso de levantamiento de la información se utilizaron fichas de observación y entrevistas semiestructuradas realizadas a grupos focales integrados por visitantes, vecinos y actores clave para establecer el uso, conocimiento e importancia de los atractivos; además de establecer el perfil del potencial visitante. Es así que, mediante la investigación y el análisis, se determinaron tres potenciales atractivos (árboles patrimoniales, fauna emblemática e historia del Parque La Alameda) y dos actividades interpretativas (función de títeres e historieta narrativa), que actúan como herramientas mediadoras entre la sociedad y su patrimonio, despertando el interés por conocer el entorno natural y cultural de una manera innovadora y creativa, además de responder a las necesidades de los visitantes.

Palabras clave: interpretación patrimonial, turismo cultural, patrimonio, emblemático, valoración de patrimonio, Parque La Alameda, Quito.

Este es un artículo Open Access bajo la licencia Creative Commons AtribuciónNoComercial-Compartirlgual 4.0

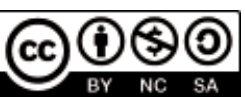




\begin{abstract}
La Alameda Park is the oldest park in the city of Quito; it is part of the heritage protection area that has a variety of tangible and intangible natural and cultural attractions of heritage and emblematic categorization that are part of the identity of the Quiteño. However, the scarce information and recreational-educational activities related to its attractions have turned it into a park of passage. For this reason, the design of an interpretive program with recreational-educational activities is a management tool for the valuation and conservation of the place and the dissemination of cultural tourism.

In the process of collecting information, observation sheets and semi-structured interviews were used with focus groups made up of visitors, neighbors and key actors to establish the use, knowledge and importance of the attractions; in addition to establishing the profile of the potential visitor. Thus, through research and analysis, three potential attractions were identified (heritage trees, emblematic fauna and the history of the Alameda Park) and two interpretive activities (puppet shows and storytelling), which act as mediating tools between society and its heritage, awakening interest in knowing the natural and cultural environment in an innovative and creative way, as well as responding to the needs of visitors.
\end{abstract}

Keywords: heritage interpretation, cultural tourism, heritage, emblematic, heritage valuation, La Alameda Park.

\title{
Introducción
}

El caso de estudio propuesto es el Parque La Alameda, ubicado en Quito, Ecuador; en una trama urbana consolidada, de gran afluencia vehicular. Este parque es poseedor de diversidad de árboles centenarios, históricos notables, singulares y emblemáticos, pertenecientes al Primer Jardín Botánico de Quito a cargo del padre Sodiro. «Fueron declarados patrimoniales por el Municipio del Distrito Metropolitano de Quito en el año 2013, según resolución municipal C-433» (Declaratoria de Árboles Patrimoniales, 2013, p. 3). También, el Parque La Alameda, alberga aves, anfibios, reptiles e insectos, algunos «declarados emblemáticos por el Municipio del Distrito Metropolitano de Quito en el año 2012 según resolución C-349, debido a su relación cotidiana con los habitantes y/o visitantes, su belleza, valor cultural y amenaza de conservación» (Declaratoria de Fauna Emblemática, 2012, p. 5).

Además, existen bienes muebles e inmuebles artísticos y arquitectónicos «que muestran a pequeña escala la diversidad estilística, estética y artística de la ciudad de Quito; algunos catalogados patrimoniales por su valor monumental, histórico, cultural y religioso. Muchos de ellos realizados por artistas: alemanes, italianos franceses y ecuatorianos» (D’Ercole y Metzger, 2002, p. 21), como el monumento a Simón Bolívar, el edificio patrimonial propiedad del Observatorio Astronómico de Quito y su Museo Astronómico.

A pesar de su «categorización de parque emblemático a escala metropolitana, parte del área de protección patrimonial» (Código Orgánico de Organización Territorial, Autonomía y Descentralización [COOTAD], 2012, p. 55) y ser promocionado como un atractivo turístico de la ciudad de Quito en páginas web del municipio y 
tripadvisor, tiene escasa información y actividades recreativas-educativas relacionadas con sus atractivos patrimoniales y emblemáticos.

Frente a esta realidad, nos planteamos como objetivo gestionar de manera innovadora y creativa el patrimonio natural y cultural del Parque La Alameda, utilizando «la interpretación patrimonial como herramienta educativa y recreativa; conjugando el disfrute con la conservación y la posibilidad de crear actividades turísticas sostenibles» (García Valecillo, 2009, p. 273); motivando tanto a los visitantes como a los anfitriones a conocer el patrimonio tangible e intangible, parte de la identidad quiteña.

Las actividades interpretativas de referencia son: (a) La Fundación Charles Darwin en Galápagos con su programa interpretativo edutenimiento -fusión de entretenimiento, educación y arte-, logrando dar a conocer en toda la provincia la historia de las islas por medio de títeres y comics que abordan temas ambientales, científicos y culturales (Monsalve, 2013); (b) El Parque Histórico Guayaquil con sus representaciones teatrales de la época cacaotera, rescatando las costumbres y tradiciones del montubio; (c) La Fundación Quito Eterno, quienes conjugan la guianza tradicional con las artes escénicas para narrar la historia de Quito desde la experiencia de los personajes históricos en espacios patrimoniales; y (d) El programa interpretativo ejecutado por el Instituto Metropolitano de Patrimonio (IMP), conocido como Los Leyendarios que mediante «representaciones de personajes de leyendas en títeres gigantes y folletos interpretativos buscan concientizar a los dueños y residentes del centro histórico del valor patrimonial que tienen las edificaciones» (2015, párr. 3).

Las actividades interpretativas ejecutadas por estas instituciones tienen por objetivo mostrar de una manera diferente, divertida e interesante temas culturales, históricos y naturales; motivando a los visitantes a conocer más del lugar. Creando reflexiones acerca de su historia, fortaleciendo la identidad cultural, «valorizando y conservando sus recursos naturales y culturales; a través de actividades interpretativas de creatividad artística y/o publicaciones interpretativas» (Bazán, 2014); conectando a la gente emocional e intelectualmente con la historia y naturaleza.

Por consiguiente, la convergencia de turismo cultural y patrimonio es inherente, ya que contribuyen al desarrollo socioeconómico de los pueblos, a mejorar las relaciones interculturales, la inclusión social, y al afianzamiento de la identidad cultural mediante programas y planes de manejo sostenibles que rehabilitan valores patrimoniales, pasados y presentes; rescatan nuevos patrimonios de forma integral naturaleza y cultura, y contribuyen con programas interpretativos y de difusión que influyen en la conservación de los patrimonios. (Fernández y Guzmán, 2002).

\section{Objetivos}

\section{Objetivo general}

Realizar un programa de interpretación patrimonial que genere valoración y conservación de los recursos naturales y culturales del Parque La Alameda. 


\section{Objetivos específicos}

- Analizar los parámetros y lineamientos del turismo cultural, patrimonio y la interpretación patrimonial.

- Determinar el estado situacional del Parque La Alameda y su entorno.

- Identificar los recursos naturales y culturales del Parque La Alameda, su estado de conservación, valoración, catalogación patrimonial y emblemática.

- Diseñar un programa interpretativo en el Parque La Alameda acorde a sus recursos naturales y culturales tangibles e intangibles.

\section{Metodología}

Utilizamos la «investigación cualitativa, buscando identificar temas, referencias, opiniones y percepciones relacionadas con el problema de estudio» (Hernández, Fernández y Baptista, 2014, p. 9); basándonos en un proceso inductivo-deductivo, de lo particular a lo general y viceversa, partiendo de la observación, recolección de datos y análisis, y generando un planteamiento teórico que permita alcanzar los objetivos.

La recolección de información la realizamos mediante la investigación de campo, investigación bibliográfica (publicaciones, ordenanzas municipales, noticias y documentación electrónica de artículos académicos), conversaciones personales con técnicos del Instituto Metropolitano de Patrimonio, Jardín Botánico de Quito, Secretaría de Ambiente, Archivo Nacional de Historia y referencias empíricas sobre interpretación patrimonial y turismo cultural. Encontramos que Tilden, en 1957 fue el primero en utilizar este tipo de metodología en el «Servicio de Parques Nacionales de los Estados Unidos a fines del siglo XIX, desarrollando actividades guiadas que favorezcan el cambio de actitudes». Entendiendo que generan experiencias recreativas, motivadoras, educativas y participativas dando un significado especial a los espacios naturales y/o culturales como una estrategia para la gestión del recurso.

Con respecto al turismo cultural, Martorell lo plantea como «turismo nacional orientado a lo cultural, altamente importante como parte de una política cultural que busque fortalecer la esencia y la identidad de una nación» (2017, p. 61). En buena cuenta, lo que no se conoce no se valora ni se respeta, por ende, no existe una valoración al patrimonio cultural.

En lo concerniente a la investigación de campo utilizamos fichas de observación directa, así como entrevistas con un cuestionario semiestructurado. Para Hernández et al. son «de manejo flexible, abierto y neutral; para que los entrevistados se sientan en confianza de contestar, con la facilidad de ser aplicado a grupos pequeños de tres o cinco personas» (2014, p. 418). Este instrumento se aplicó entre los meses de mayo a setiembre de 2015, entre semana y fines de semana, en diversos horarios y fechas no consecutivas, a varios grupos focales entre 15 y 65 años de edad, conformados por visitantes, vecinos, estudiantes, profesores y actores clave como la Sra. Patricia González, presidenta de la Asociación de comerciantes de la Alameda; el Sr. Danilo Rivera, quien presta el servicio de alquiler de botes; la Srta. Ma. Eugenia Ramos, encargada del Observatorio Astronómico de Quito y museo astronómico; el P. Emilio Raza, párroco de la Iglesia del Belén; logrando tener un intercambio de información interesante. 
Tanto la investigación de campo como la bibliográfica nos permitieron contar con datos cuantitativos en edad, sexo y residencia; y cualitativos en identidad, pertenencia e importancia local; necesarios para plantear soluciones frente al escaso conocimiento de los recursos naturales y culturales con catalogación patrimonial y emblemática que tiene el Parque La Alameda.

\section{Resultados}

Luego de realizar la observación directa y las entrevistas con el cuestionario semiestructurado a 45 grupos focales (Apéndices 1 y 2) se logró diagnosticar tres grupos de potenciales atractivos turísticos que pueden ser mejorados a través de la interpretación patrimonial para facilitar la «experiencia turística» (Navarro, 2015, p. 354), y determinar las actividades recreativas-educativas idóneas.

El análisis de las entrevistas determinó que es un espacio urbano de uso cotidiano con afluencia de usuarios acompañados de familia o amigos, en su mayoría del sur y centro del Distrito Metropolitano de Quito, quienes realizan actividades recreativas y culturales relacionadas en un $70 \%$ con los atractivos tradicionales del parque como el Observatorio Astronómico de Quito y museo astronómico, el Churo de la Alameda y las comidas y bebidas típicas.

Además, el 94\% de los usuarios señalaron que existe limitada información acerca de otros atractivos del parque como historia de La Alameda, fauna, árboles patrimoniales y monumentos de personajes históricos. El $40 \%$ de los entrevistados opinaron que es muy importante conocer estos atractivos por cultura general e identidad quiteña, pero de una manera diferente y entretenida. De la lista de actividades recreativas-educativas formuladas eligieron función de títeres e historieta narrativa descriptiva con pequeñas actividades lúdicas.

\section{Datos generales}

- Los usuarios del Parque La Alameda, en este estudio, son 52\% de sexo masculino y 8\% de sexo femenino. En cuanto la edad, predominan los de 30 a 44 años (38\%) y de 15 a 29 años (31\%), según se muestra en la Figura 1.

\section{Figura 1}

Sexo y edad de los usuarios del Parque La Alameda

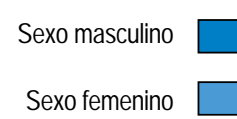


- $\quad$ Residencia. El 37\% de usuarios del Parque La Alameda proviene del sector sur, 34\% del sector centro y un porcentaje relativamente menor proviene del norte del Distrito Metropolitano de Quito. Demostrando que es un parque de uso ciudadano sin preferencia sectorial (Figura 2).

\section{Figura 2}

Residencia de los usuarios del Parque La Alameda

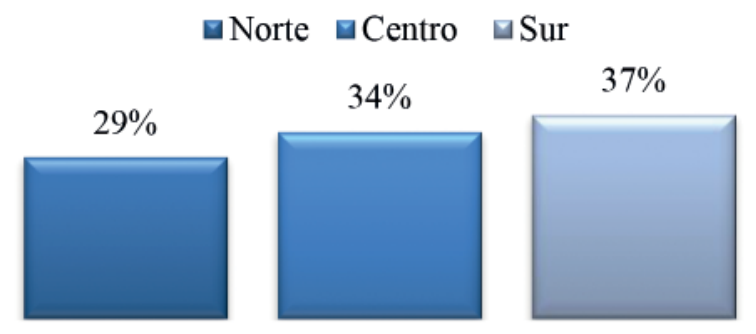

\section{Actividades}

Dado que el Parque La Alameda se ubica en una zona comercial e institucional la frecuencia de visita de los usuarios varía entre y fines de semana. Entre semana el 25\% de usuarios del parque son estudiantes primarios que realizan actividades recreativas, educativas y culturales. Solo un $9 \%$ de este grupo realiza algún tipo de actividad el fin de semana.

La frecuencia de visita los fines de semana es como sigue: 15\% amas de casa y $12 \%$ profesionales; quienes realizan actividades recreativas y deportivas acompañados de sus hijos u otros familiares de 8 a 12 años de edad.

- Frecuencia de visitas y ocupación. El 10\% de las visitas mensuales corresponde a usuarios esporádicos que realizan actividades de descanso, abastecimiento de agua del ciclo paseo o extranjeros que visitan el observatorio y museo (Figura 3).

\section{Figura 3}

Frecuencia de visita y ocupación del Parque La Alameda

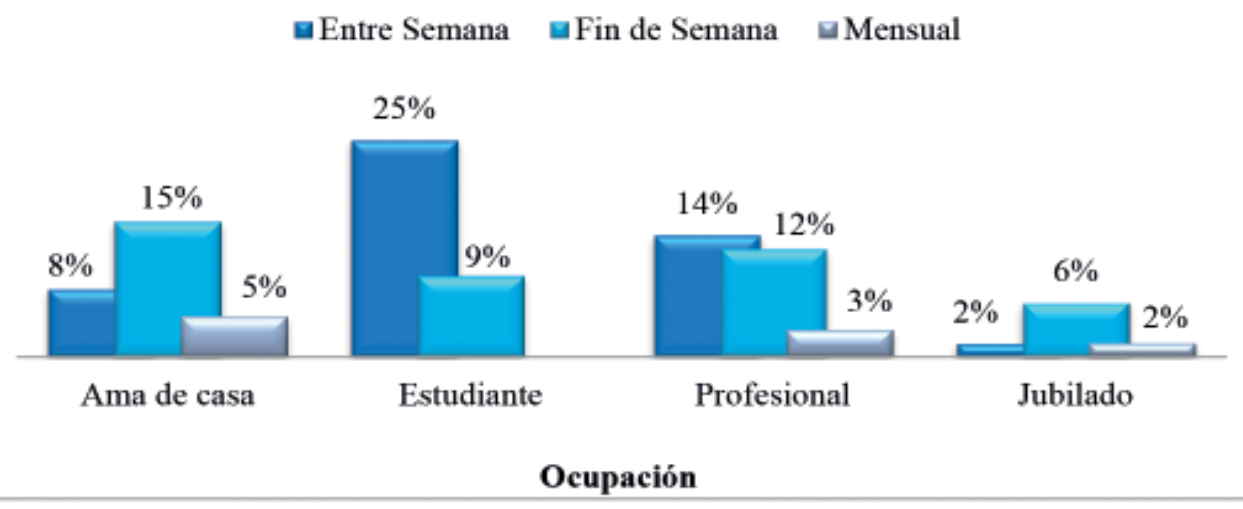


- Actividades de ocio, recreación y cultura. Según se muestra en el Figura 4, la actividad más disfrutada sin discriminación de hora, día, sexo y edad es el paseo en bote; la visita al observatorio y museo la realizan en su mayoría estudiantes de primaria, entre semana, según el horario del museo. Cabe anotar que las personas entrevistadas los fines de semana no tienen mayor conocimiento del horario de atención del museo y los servicios que presta el observatorio.

\section{Figura 4}

Actividades de ocio, recreación y cultura

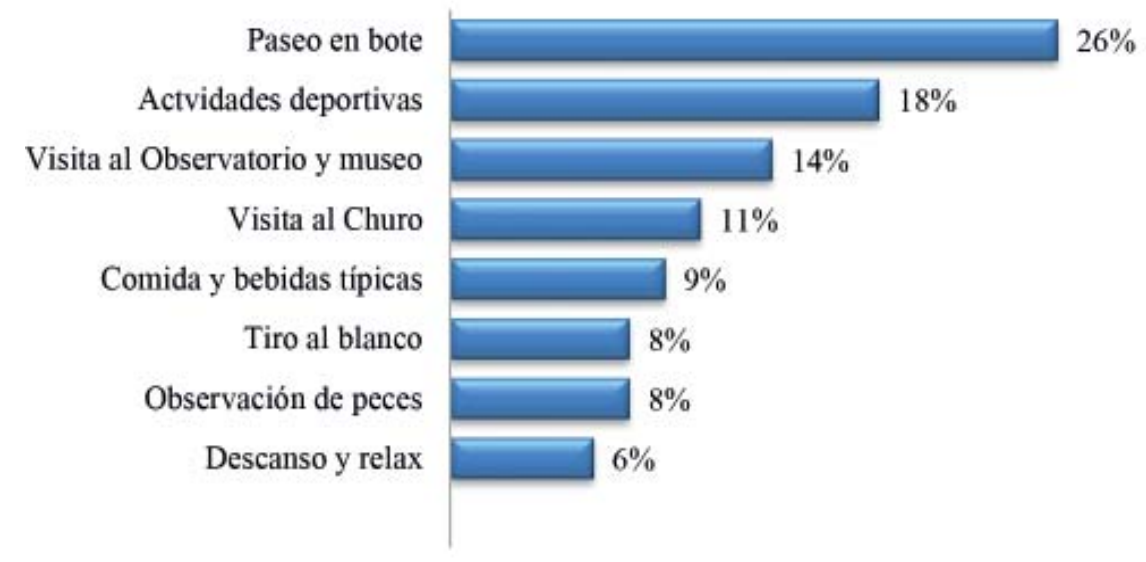

\section{Atractivos naturales y culturales}

- Atractivos del Parque La Alameda. Para 31\% de los entrevistados la laguna es el principal atractivo por los paseos en bote y para 23\% por ser el primer mirador de Quito; 18\% entre ellos estudiantes, profesionales y amas de casa, mencionaron los árboles patrimoniales, sin embargo, poco sabían la ubicación o nombres comunes; $12 \%$ de los usuarios indicaron las comidas típicas y bebidas, de interés a quienes les encantan «las cosas finas» y son clientes fijos de sus «caseritas» (Figura 5).

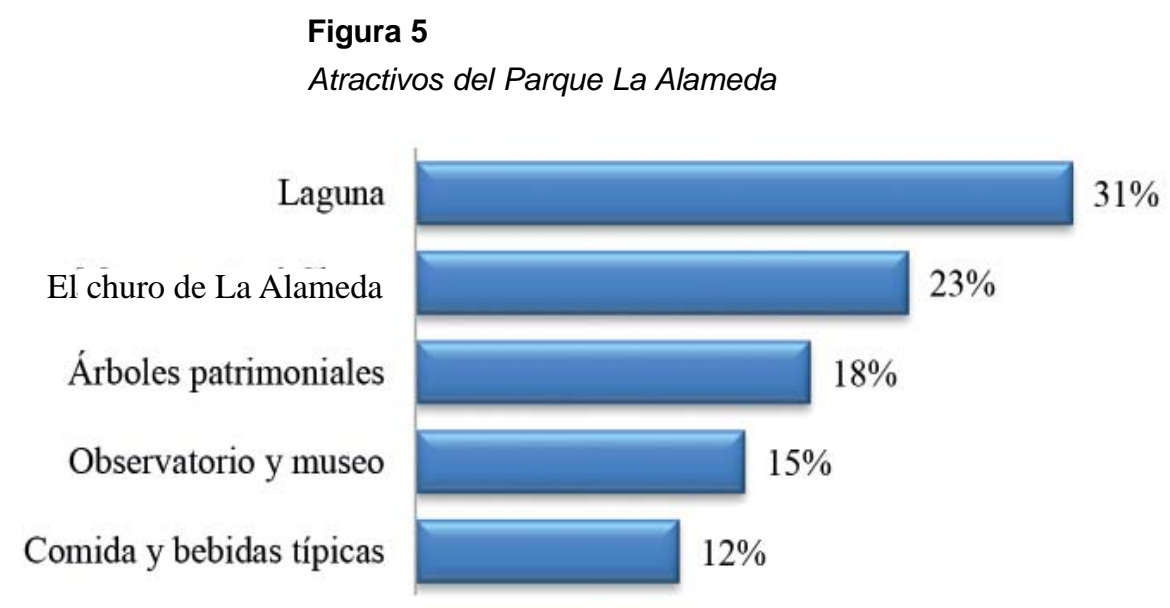


- Atractivos patrimoniales y emblemáticos. Los usuarios respondieron que los atractivos conocidos con declaración patrimonial son el Observatorio Astronómico de Quito y museo (46\%) y los árboles patrimoniales (35\%). Además la opinión de los usuarios frecuentes sugiere que todos los atractivos del Parque La Alameda son emblemáticos, destacándose la laguna y el Churo de la Alameda. No obstante, no conocían que existen árboles y animales emblemáticos con declaratoria (Figura 6).

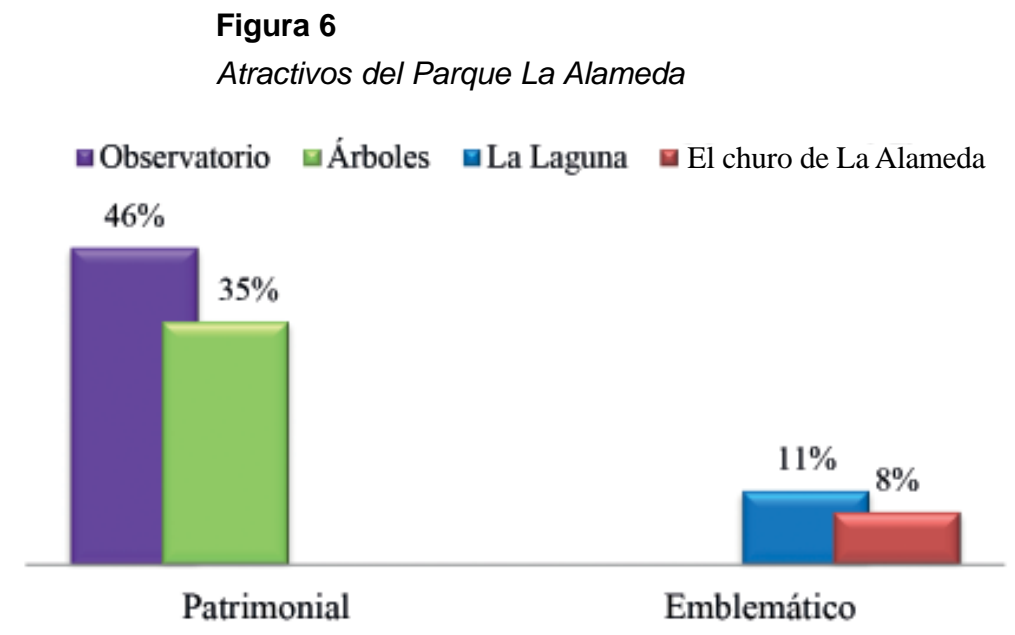

- Información de los atractivos. El 94\% de los usuarios considera que existe información relevante respecto de la historia de La Alameda 37\%, con los árboles patrimoniales 26\%; con la fauna 23\%, y un pequeño porcentaje con los Monumentos a personajes históricos (Figura 7).

Figura 7

Información de los atractivos

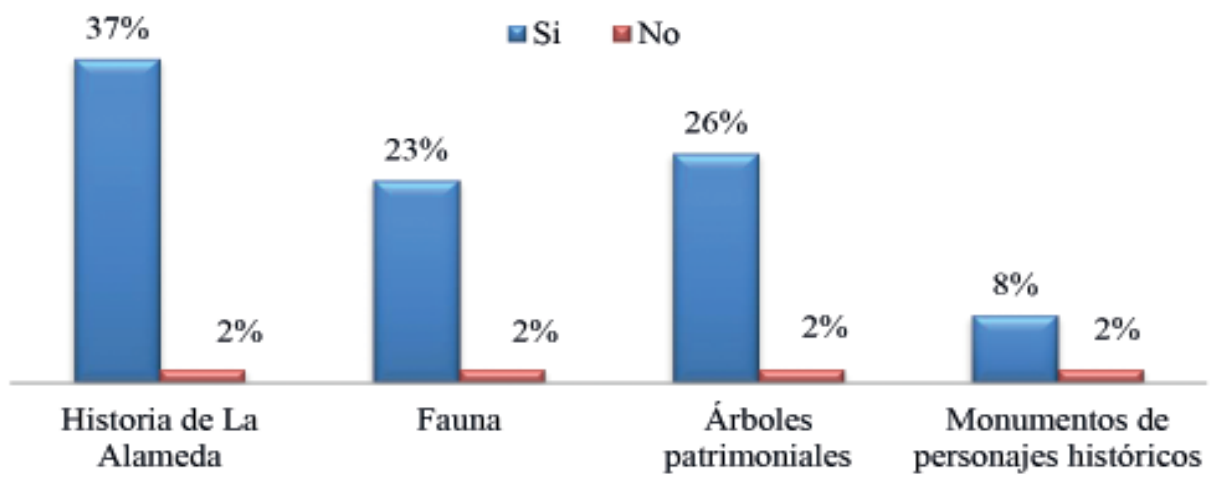




\section{Valoración cualitativa de los atractivos}

En la valoración se tomaron en cuenta los atractivos conocidos y poco conocidos aplicando una escala cualitativa de: muy importante, importante, poco importante y nada importante.

- Importancia del conocimiento de los atractivos

Los usuarios consideraron que es muy importante (15\%) conocer la historia de La Alameda, teniendo en cuenta que tiene un observatorio muy antiguo. Cabe precisar que se conoce mucho más del Centro Histórico de Quito.

Consideran importante (13\%) tener información sobre los árboles patrimoniales, la fauna, en especial, de las aves que allí habitan, pues no se cuenta con información; mencionan que en ocasiones han observado colibríes y otras especies. Opinan que les parece un poco extraño encontrar animales en un parque rodeado de buses, automóviles y edificios.

La fiesta de la Virgen del Quinche, los monumentos a los personajes históricos y Churo de la Alameda despiertan poco interés en los usuarios.

El 40\% de los entrevistados considera importante conocer los atractivos culturales y naturales del parque por identidad quiteña, y hacer uso de los servicios que ofrece un parque emblemático antiguo de paso (Figura 8).

Figura 8

Importancia del conocimiento de los atractivos

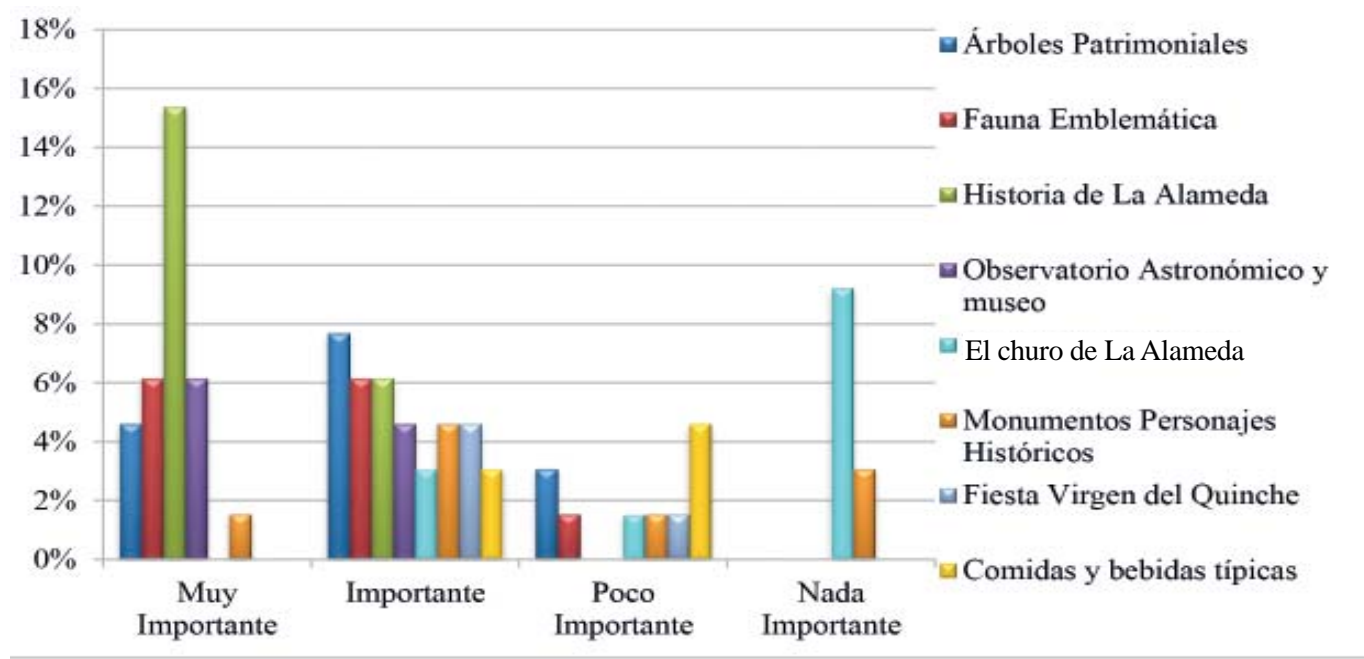




\section{Programa de interpretación patrimonial}

- $\quad$ Selección de medios interpretativos

Para la selección de los atractivos se solicitó a los grupos focales que elijan entre varias actividades interpretativas planteadas en la entrevista, ya sea títeres, dramatizaciones, historietas, guianza y sendero con señalética. La formulación de las actividades se realizó sobre la base de los atractivos naturales y culturales del parque, los grupos de familiares, amigos, y estudiantes -en su mayoría de primaria- que visitan el observatorio y museo.

Optaron 34\% por la función de títeres y 26\% por la historieta narrativa descriptiva. Además, se clarificó el perfil del usuario, el conocimiento e importancia de los atractivos culturales y naturales del parque, desde la apreciación de sus usuarios frecuentes entre y/o fines de semana; considerando al Parque La Alameda como un lugar que guarda historia la cual debe ser conocida por cultura general e identidad (Figura 9).

Figura 9

Medios interpretativos

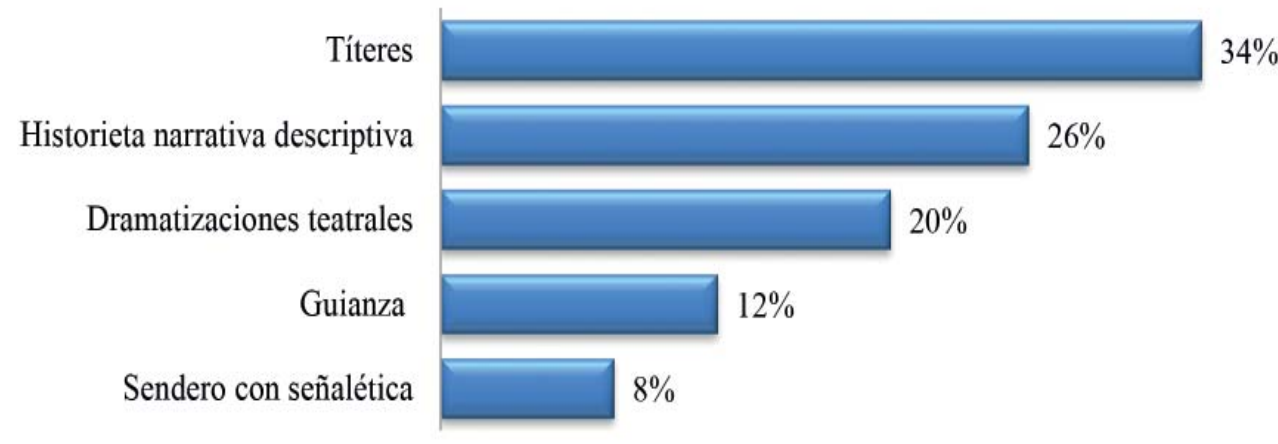

\section{Clasificación de los atractivos naturales y culturales del Parque La Alameda}

La clasificación la realizamos sobre la base de la información sobre el aspecto formal tangible e intangible y el aspecto funcional; valores intrínsecos o características relevantes del recurso turístico que provocan una visita (Navarro, 2015) con la posibilidad de ser convertidos en atractivos.

Para la categorización tomamos en cuenta los atractivos más conocidos y frecuentados; agrupándolos según el criterio que propone Navarro, en el que se «relaciona la clasificación de Boullón con la del patrimonio de la Unesco» (2015, p. 345).

- Clasificación de los atractivos naturales y culturales

La ventaja de esta clasificación es considerar a la materialidad como una asociación entre lo intangible y lo tangible de los recursos turísticos, con el propósito de mejorar o crear atractivos turísticos interesantes que 
tengan un vínculo sensorial, intelectual y vivencial, favoreciendo el contacto y satisfaciendo las necesidades motivacionales de los visitantes o turistas (Navarro, 2015). El conocimiento de la materialidad ayuda a crear atractivos tangibles de la intangibilidad y a identificar recursos tangibles relevantes que ameriten ser conocidos y valorados.

Tabla 1

Clasificación de los atractivos naturales y culturales

\begin{tabular}{|c|c|c|c|c|}
\hline $\begin{array}{l}\text { NOMBRE DEL } \\
\text { ATRACTIVO }\end{array}$ & CATEGORÍA & MATERIALIDAD & TIPO & SUBTIPO \\
\hline $\begin{array}{l}\text { Árboles } \\
\text { patrimoniales }\end{array}$ & Sitios naturales & Tangible & $\begin{array}{c}\text { Lugares de } \\
\text { observación de flora }\end{array}$ & $\begin{array}{c}\text { Árboles monumentales } \\
\text { e históricos }\end{array}$ \\
\hline $\begin{array}{l}\text { Fauna } \\
\text { emblemática }\end{array}$ & Sitios naturales & Tangible & $\begin{array}{c}\text { Lugares de } \\
\text { observación de fauna }\end{array}$ & $\begin{array}{l}\text { Aves urbanas, } \\
\text { reptiles y anfibios }\end{array}$ \\
\hline $\begin{array}{l}\text { Observatorio } \\
\text { astronómico y } \\
\text { museo }\end{array}$ & $\begin{array}{l}\text { Manifestaciones } \\
\text { culturales }\end{array}$ & Tangible, intangible & Lugar histórico & $\begin{array}{c}\text { Observatorio, museo y } \\
\text { biblioteca }\end{array}$ \\
\hline $\begin{array}{l}\text { Churo de } \\
\text { La Alameda }\end{array}$ & $\begin{array}{l}\text { Manifestaciones } \\
\text { culturales }\end{array}$ & Tangible, inmueble & Lugar histórico & Mirador \\
\hline $\begin{array}{l}\text { Monumentos de } \\
\text { personajes históricos }\end{array}$ & $\begin{array}{l}\text { Manifestaciones } \\
\text { culturales }\end{array}$ & Tangible, mueble & Arte público & Esculturas \\
\hline $\begin{array}{l}\text { Fiesta a la Virgen } \\
\text { del Quinche }\end{array}$ & $\begin{array}{l}\text { Acontecimientos } \\
\text { programados }\end{array}$ & Intangible, tangible & Fiestas & Fiestas Religiosas \\
\hline $\begin{array}{l}\text { Comida y bebidas } \\
\text { típicas }\end{array}$ & Folklore & Intangible, tangible & Gastronomía & Platos tradicionales \\
\hline $\begin{array}{l}\text { Historia de } \\
\text { La Alameda }\end{array}$ & Folklore & Intangible & Herencia cultural & $\begin{array}{l}\text { Acontecimientos } \\
\text { históricos }\end{array}$ \\
\hline
\end{tabular}

\section{Valoración cuantitativa de los atractivos turísticos del Parque La Alameda}

La valoración de los atractivos se realizó de acuerdo a la metodología del Plan Q 2002 que considera cinco criterios: unicidad, notoriedad, valor intrínseco, concentración de la oferta y carácter quiteño, cada uno con un valor relacionado con su importancia; siendo el puntaje máximo de 5 y el mínimo de 1 . Los criterios de mayor peso son: Unicidad, Valor intrínseco y Notoriedad, que generan el mayor deseo de conocerlos (Corporación Metropolitana de Turismo de Quito, 2002, p. 39). Además, está valoración ayuda a determinar los atractivos turísticos con alto valor intrínseco y carácter quiteño, considerando que son atractivos locales de ocio y recreación con escasas actividades educativo-recreativas.

Para el levantamiento de criterios de valoración se utilizó información bibliográfica, fichas de observación, entrevistas y conversaciones con el personal técnico del Instituto Metropolitano de Patrimonio, Asociación de Arboricultura y Archivo de Historia. 
En el análisis y estudio de los atractivos resultó con criterios de mayor peso, con valoración 4: árboles patrimoniales, fauna emblemática y observatorio astronómico y museo por sus características científicas, técnicas y de relación cotidiana con los vecinos y visitantes del parque.

A pesar de que el atractivo intangible Historia de La Alameda tiene una valoración 2 en Unicidad y 1 en Notoriedad, puede ser considerado potencial atractivo por su Valor intrínseco de 3 y Carácter quiteño 4.

Por otra parte, la puntuación 3 de Concentración de la oferta para todos los atractivos analizados, es consecuencia de la ubicación en la zona turística Centro abastecido de diversidad de servicios de alojamiento y restauración pero con escasas actividades turísticas, recreativas y/o culturales. Existiendo mayor concentración de estas actividades en las zonas Centro Histórico y Mariscal. A esto, se suma que la notoriedad, el grado de conocimiento, que tiene un valor de 1 o 2, resultado de la escasa información turística y actividades educativasrecreativas esporádicas relacionadas solo con el Observatorio Astronómico de Quito.

De acuerdo a la relación de las cinco variables determinamos los recursos relevantes a ser optimizados o convertidos en atractivos turísticos que mejoran la relación de contacto atractivo visitante y/o vecino (Figura 10).

Figura 10

Criterios de la valoración de los atractivos

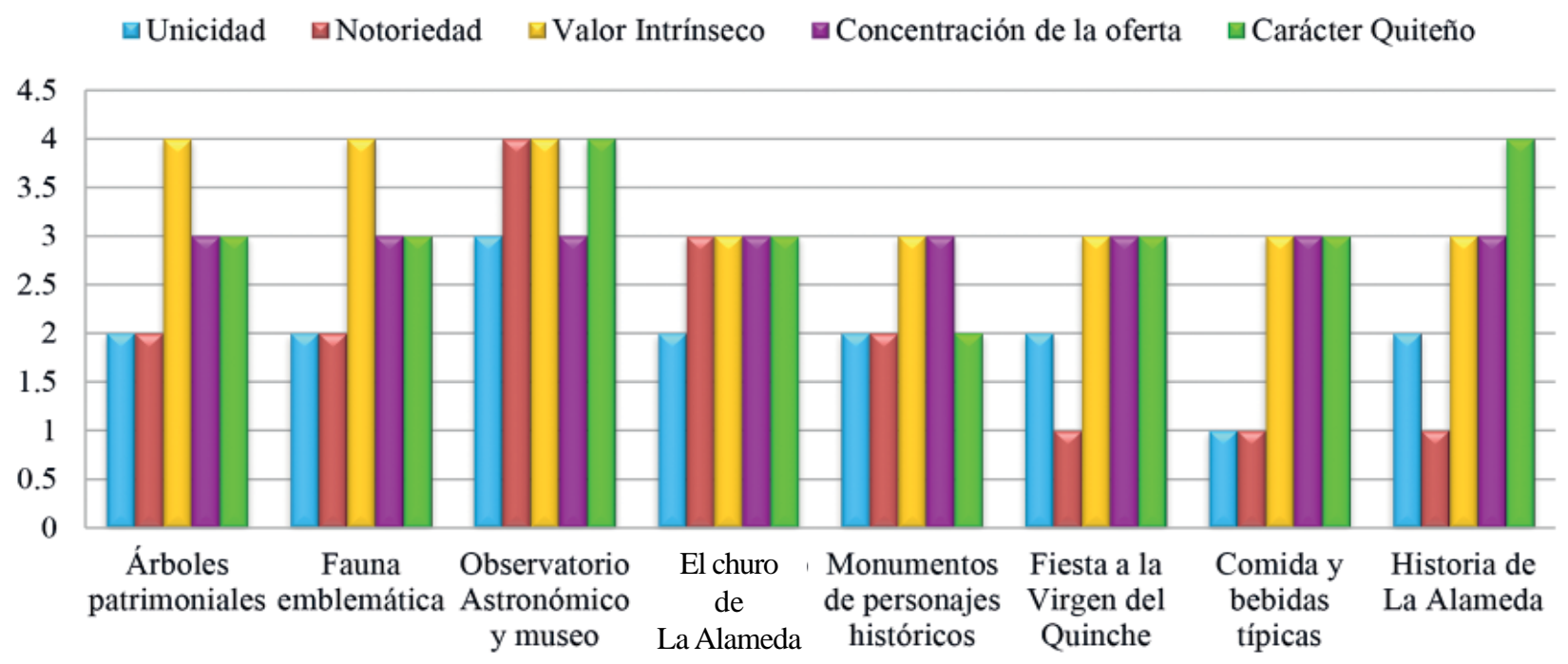




\section{Selección de atractivos y mensajes}

En el proceso de selección de los potenciales atractivos influyó la clasificación y valoración de los atractivos, datos obtenidos de las fichas de observación y de la entrevista semiestructurada. Es decir, el mayor deseo de ser conocidos de valoración 4 a 5, el Carácter quiteño, relacionado específicamente con la identidad local y el Valor intrínseco, información técnica, científica e histórica.

Este análisis arrogó dos de categoría sitios naturales y uno de folklore, intangible de subtipo acontecimientos históricos.

- Árboles patrimoniales

- Fauna emblemática

- Historia de La Alameda

El análisis nos permitió seleccionar atractivos tangibles e intangibles de importancia local, vinculados con eventos históricos y narraciones poco conocidos, pero que deben ser gestionados y difundidos a través de la interpretación patrimonial para generar un vínculo intelectual y/o sensorial que motive al quiteño o visitante al conocimiento y disfrute de los atractivos del Parque La Alameda de una manera diferente, divertida y amena, e impulsarlo como un atractivo turístico local de interés cultural.

\section{Conclusiones}

- La interpretación es una herramienta de gestión y difusión tanto para el patrimonio como para el turismo, pues permite que los recursos naturales y culturales -materia prima-, se conviertan en atractivos de interés y visitables, por medio de infraestructuras y/o actividades interpretativas que motiven el respeto, el aprecio y contribuyan a la conservación y valoración de un lugar visitado. El diseñar un programa interpretativo en el Parque La Alameda con actividades de creatividad artística y medios impresos, obras de títeres e historietas facilita el conocimiento, el contacto con los atractivos y despierta el interés del público visitante.

- El análisis del entorno del Parque La Alameda nos conlleva a la confirmación de que es un parque emblemático de ocio y recreación de uso local y nacional. Gestionado estética, arquitectónica y funcionalmente por entidades públicas (Administración Zonal Centro, Instituto Metropolitano de Patrimonio, Unidad de Gestión de Espacio Público y Escuela Politécnica Nacional) y privadas (Asociación de Comerciantes de La Alameda y vecinos) interesados en impulsar la implementación y ejecución de un programa interpretativo.

- Al realizar el diagnóstico de los atractivos encontramos ocho atractivos entre naturales y culturales con criterios de valoración alta y media; para este proyecto se escogieron tres atractivos: historia de La Alameda, fauna emblemática y árboles patrimoniales en respuesta a las necesidades culturales, educativas y recreativas del público objetivo que visita el Parque La Alameda. 
- Mediante las entrevistas determinamos un segmento de mercado local de familias y amigos, los fines de semana; y estudiantes de $4 .^{\circ}$ a $7 .^{\circ}$ año de básica, con intereses educativos, culturales y recreativos, entre semana. Grupos a los que irán dirigidas las actividades interpretativas.

- $\quad$ Para el diseño del programa interpretativo consideramos el segmento de mercado, las respuestas de los entrevistados y los mensajes interpretativos a ser transmitidos. Así tenemos las actividades interpretativas con mayor aceptación por los visitantes: función de títeres 34\% e historieta 26\%; a lo que se sumó la factibilidad de implementación en un espacio de uso público y recreativo y la facilidad de ser ejecutadas en forma independiente o en conjunto dependiendo de las necesidades de la audiencia presente.

- La función de títeres y la historieta son actividades interpretativas educativas-recreativas que al desarrollarse dentro del Municipio del Distrito Metropolitano de Quito necesitan un análisis y aprobación de la Unidad de Cultura de la Administración Zonal Centro para ser implementadas y ejecutadas tanto para tramitar el permiso de uso del espacio público, como para los respectivos auspicios con el Instituto Metropolitano de Patrimonio. 


\section{Referencias}

Bazán, H. (2014). La interpretación del patrimonio geomorfológico en los picos de Europa: una propuesta para su aprovechamiento didáctico y geoturístico [tesis doctoral no publicada, Universidad de Valladolid]. España.

Código Orgánico de Organización Territorial, Autonomía y Descentralización. (2012). Resolución 171/2012. http://quitoambiente.gob.ec/ ambiente/index.php/biblioteca-digital/category/15-marco-normativo

Corporación Metropolitana de Turismo de Quito. (2002). Plan Estratégico de Turismo de la CMT: Fase I Análisis. Quito.

Declaratoria de Árboles Patrimoniales (2013). Resolución C433/2013. http://www7.quito.gob.ec/mdmq_ordenanzas/ Resoluciones\%20de\%20Concejo/A\%C3\%B10\%20\%202013/RC-2013-433\%20-\%20\%C3\%81RBOLES\%20PATRIMONIALES.pdf

Declaratoria de Fauna Emblemática. (2012). Resolución C349/2012. http://www7.quito.gob.ec/mdmq_ordenanzas/Resoluciones\%20 de\%20Concejo/A\%C3\%B10\%20\%202012/RC-2012-349\%20-\%20FAUNA\%20EMBLEM\% C3\%81TICA\%20DE\%20QUITO\%20\%20DECLARATORIA.pdf

D’Ercole, R. y Metzger, P. (2002). El patrimonio en el Distrito Metropolitano de Quito: valoración de sus principales elementos y análisis espacial. Programa de investigación «sistema de información y riesgos». Quito. Institut de Recherche pour le Développement y MDMQ.

García Valecillo, Z. (2009). ¿Cómo acercar los bienes patrimoniales a los ciudadanos? Revista de Turismo y Patrimonio Cultural. http://www.pasosonline.org/Publicados/7209/PS0209_9.pdf

Hernández, R., Fernández, C. y Baptista, P. (2014). Metodología de la Investigación (5. ed.). México: McGraw Hill.

Instituto Metropolitano de Patrimonio. (2015). Manual de Conservación del Patrimonio Edificado. http://www.patrimonio.quito.gob.ec/ index.php/difusion/actualidad/283-el-instituto-metropolitano-de-patrimonio-presento-el-manual-de-conservacion-del-patrimonio-edificado

Monsalve, G. (2013). Ciencia y medioambiente con el teatro de las sombras. Noticias Fundación Charles Darwin. http:// test.darwinfoundation.org/es/noticias/2013/04/22/teatro-de-sombra/

Martorell, A. (2017). Turismo cultural. Reflexiones para un encuentro sostenible entre turismo y cultura. Lima: Fondo Editorial. USMP.

Navarro, D. (2015). Recursos turísticos y atractivos turísticos: conceptualización, clasificación y valoración. Cuadernos de Turismo, (35), 335 -357. doi: 10.6018/turismo.35.221641

Pozo, J. (2002). Plan especial La Alameda. Quito: MDMQ.

Tilden, F. (1957). Interpreting our heritage. Carolina: Press the University of North Carolina.

Alexandra Araguillín Rojas

Pontificia Universidad Católica del Ecuador

Consultor de proyectos turísticos especializado en temas de conservación patrimonial, ambiental y salud.

adaraguillinr@gmail.com

ORCID: https://orcid.org/0000-0002-9724-9843

Renato Gustavo Cevallos Becerra

Universidad UTE, Quito, Ecuador

Docente a tiempo parcial desde el año 2008, especializado en Turismo Sostenible y Gestión de Destinos Turísticos.

renato.cevallos@ute.edu.ec

ORCID: https://orcid.org/0000-0002-5515-1143 


\section{Apéndice 1. Entrevista semiestructurada \\ PROGRAMA DE INTERPRETACIÓN PATRIMONIAL EN EL PARQUE LA ALAMEDA DE QUITO}

Cuestionario comunitario

Esta encuesta tiene por finalidad determinar los atractivos naturales y culturales del Parque La Alameda de Quito, para promover su valoración y conservación a través de un programa de interpretación patrimonial.

\begin{tabular}{|c|c|c|c|c|c|c|}
\hline \multicolumn{7}{|c|}{ 1. Datos generales } \\
\hline Sexo: & $\mathrm{F}$ & $\mathrm{M}$ & Edad: & & Lugar de residencia: & \\
\hline Ocupación: & \multicolumn{3}{|c|}{ Ama de casa } & Estudiante & Profesional & Jubilado \\
\hline \multicolumn{7}{|c|}{ Nombre de la persona entrevistada: } \\
\hline
\end{tabular}

\begin{tabular}{l|l}
\hline \multirow{2}{*}{ ¿Con que frecuencia viene al Parque La Alameda? } & Entre semana \\
\cline { 2 - 2 } & Fin de semana \\
\cline { 2 - 2 } ¿Con quién vino al Parque La Alameda? & Familia \\
\cline { 2 - 2 } & Amigos \\
\cline { 2 - 2 } & Solo/a \\
\hline
\end{tabular}

¿Qué tipo de actividades realiza en el parque? (ocio, recreación, deporte, cultural, especificar)

\section{Atractivos naturales y culturales}

¿Qué atractivos tiene el Parque La Alameda?

¿De los nombrados conoce alguno con declaratoria patrimonial o emblemática?

¿Cree usted que existe más información poco conocida acerca del Parque La Alameda? (especificar)

\begin{tabular}{l|l|l|l|l}
\hline 4. Valoración de los atractivos & $\begin{array}{c}\text { muy } \\
\text { importante }\end{array}$ & Importante & $\begin{array}{c}\text { poco } \\
\text { importante }\end{array}$ & $\begin{array}{c}\text { nada } \\
\text { importante }\end{array}$ \\
\hline ¿Qué atractivos considera importante conocer? & & & & \\
\hline Árboles patrimoniales & & & & \\
\hline Fauna emblemática & & & & \\
\hline Historia de La Alameda & & & & \\
\hline Observatorio astronómico y museo & & & & \\
\hline Churo de La Alameda & & & & \\
\hline Monumentos personajes & & & & \\
\hline Fiesta Virgen del Quinche & & & & \\
\hline Comidas y bebidas típicas & & & \\
\hline
\end{tabular}

\section{Programa de interpretación patrimonial}

¿Qué actividad usted considera relevante para promover la valorización y conservación de los recursos naturales y culturales del Parque La Alameda?

\begin{tabular}{|l} 
Títeres \\
\hline Dramatizaciones teatrales \\
\hline Folletos (historieta) \\
\hline Guianza \\
\hline Sendero con señalética \\
\hline
\end{tabular}




\section{Apéndice 2. Ficha de observación \\ PROGRAMA DE INTERPRETACIÓN PATRIMONIAL \\ EN EL PARQUE LA ALAMEDA, QUITO \\ IDENTIFICACIÓN DE LOS RECURSOS NATURALES Y CULTURALES \\ FICHA DE OBSERVACIÓN}

Nota: La aplicación de esta ficha de observación tiene la finalidad de recoger datos sobre los recursos naturales y culturales del Parque La Alameda en Quito para promover la conservación de los recursos naturales y culturales a través de un programa de interpretación patrimonial.

Fecha:

Hora:

Responsable:

Firma:

\section{Datos generales}

\begin{tabular}{l}
\hline Nombre del parque: \\
\hline Dirección: \\
\hline Sector:
\end{tabular}

\section{Recursos naturales}

\begin{tabular}{|c|c|c|c|c|}
\hline \multicolumn{5}{|c|}{ Flora y/o Fauna } \\
\hline Especie & N. común & Ubicación & n. ${ }^{\circ}$ & Catalogación \\
\hline & & & & \\
\hline & & & & \\
\hline & & & & \\
\hline & & & & \\
\hline & & & & \\
\hline & & & & \\
\hline & & & & \\
\hline & & & & \\
\hline & & & & \\
\hline
\end{tabular}

\section{Recursos culturales}

Bienes artísticos y/o arquitectónicos

\begin{tabular}{|c|c|c|c|c|c|c|}
\hline \multirow{2}{*}{ Elemento } & \multicolumn{3}{|c|}{ Estado de conservación } & \multirow{2}{*}{ Movilidad } & \multirow{2}{*}{ Función } & \multirow{2}{*}{ Ubicación } \\
\hline & excelente & bueno & regular & & & \\
\hline & & & & & & \\
\hline & & & & & & \\
\hline & & & & & & \\
\hline & & & & & & \\
\hline & & & & & & \\
\hline & & & & & & \\
\hline & & & & & & \\
\hline & & & & & & \\
\hline & & & & & & \\
\hline
\end{tabular}




\section{Ocupación del espacio público}

\begin{tabular}{|c|c|c|c|c|c|c|}
\hline \multirow[b]{2}{*}{ Actividades } & \multicolumn{4}{|c|}{ Edades de las personas que realizan las actividades } & \multicolumn{2}{|c|}{ Frecuencia de visita } \\
\hline & Niños & Jóvenes & Adultos & $\begin{array}{c}\text { Adultos } \\
\text { mayores }\end{array}$ & Entre semana & Fines de semana \\
\hline \multicolumn{7}{|l|}{ Paseo en bote } \\
\hline \multicolumn{7}{|c|}{ Actividades deportivas } \\
\hline \multicolumn{7}{|c|}{ Visita al Observatorio y museo } \\
\hline \multicolumn{7}{|l|}{ Visita al Churo } \\
\hline \multicolumn{7}{|c|}{ Comida y bebidas típicas } \\
\hline \multicolumn{7}{|l|}{ Tiro al blanco } \\
\hline \multicolumn{7}{|c|}{ Observación de peces } \\
\hline Descanso y relax & & & & & & \\
\hline
\end{tabular}

4. Imagen del espacio público

\begin{tabular}{l|l|l|l|l}
\hline Limpieza de las áreas verdes y/o recreativas & Sí & NO & \\
\hline Limpieza de la laguna & Sí & & NO & \\
\hline Seguridad con guardias y/o policía nacional & Sí & NO & \\
\hline Vandalismo y/o graffitis & Sí & NO & \\
\hline Presencia de indigentes y/o alcohólicos & Sí & & NO & \\
\hline
\end{tabular}

Comentarios: 